\title{
A Collaborative Group Decision Model for Internet Public Opinion Emergency with Interval Value
}

\author{
Qiansheng Zhang* \\ School of Mathematics and Statistics, Guangdong University of Foreign Studies, Guangzhou 510006, China \\ *Corresponding author: zhqiansh01@126.com \\ Received May 05, 2019; Revised June 16, 2019; Accepted July 02, 2019
}

\begin{abstract}
This paper proposes a collaborative group decision model for internet public opinion emergency with interval-valued index involved multiple decision-makers. By using the proposed emergency decision model the optimal weight of each individual and collaborative index of emergency can be determined. Finally, the comprehensive crisis of each emergency can be calculated by integrating the individual severity with the collaborative severity. According to the ranking of crisis the emergency department can make effective decision scheme to deal with all the internet public opinion emergencies.
\end{abstract}

Keywords: internet public opinion emergency, interval linguistic value, collaborative index, emergency group decision

Cite This Article: Qiansheng Zhang, "A Collaborative Group Decision Model for Internet Public Opinion Emergency with Interval Value." American Journal of Modeling and Optimization, vol. 7, no. 1 (2019): 14-19. doi: 10.12691/ajmo-7-1-3.

\section{Introduction}

Recently, the complicated internet public opinion, social emotion and attitude have triggered many unconventional emergencies. This type of internet public opinion emergency greatly impairs the harmony and stability of society [1,2]. It is urgent to make some effective decision mechanisms to deal with Internet Public Opinion Emergency (IPOE).

Due to time pressure and incomplete expert experience, it is very difficult to collect the accurate public opinion information or precise index data for IPOE in emergency decision situation. Generally, in the uncertain emergency decision environment the emergency experts usually employ interval values $[3,4]$ or interval linguistic terms $[5,6]$ to evaluate the indicators of internet public opinion emergency. Although many scholars have put forward some decision models for unconventional emergencies $[7,8,9]$, they are not suitable for dealing with uncertain emergency decision problems. In uncertain emergency decision environment, $\mathrm{Wu}$ [10] presented a fuzzy multi-criteria emergency decision-making method, Zhang [11] recently proposed a hesitant group emergency decision-making method based on prospect theory, Jing [12] presented an emergency decision-making method based on interval preference relations. Tang [13] presented a uncertain group decision model of extreme flood disaster emergency.

However, there is few works on interval-valued fuzzy collaborative decision for internet public opinion emergency. Since interval-valued fuzzy set $[14,15]$ is very applicable to assess emergency indexes and interval linguistic term [16] is suitable to model the vagueness in public opinion and emergency decision knowledge, this paper presents a collaborative decision making model for internet public opinion emergency based on interval value or linguistic interval value. As we know, internet public opinion emergency can be assessed by multiple emergency experts from different emergency sections $[17,18]$. And the internet public opinion emergency is usually evaluated by some uncertain individual indexes and collaborative indexes. Hence, it is necessary to integrate all the emergency departments to determine the interval weights of emergency experts and evaluate the individual and collaborative severity of internet public opinion emergency.

Therefore, in this paper we develop an interval fuzzy collaborative group decision approach regarding all the individual and collaborative indexes with interval value or interval linguistic terms. By combining the individual index and collaborative index of uncertain IPOE, we can calculate the overall severity of each IPOE and rank the overall severity of all the internet public opinion emergencies (IPOEs). Then the related emergency department can implement the emergency solution and make effective decision scheme to deal with most severe internet public opinion emergencies in accordance with the severity order of IPOEs.

\section{Preliminaries}

For convenience of analysis, we first introduce some basic concepts and operators of interval-values and interval linguistic terms in this section. 
Definition 1 [5] A finite and totally ordered discrete set $S=\left\{s_{i} / i=0,1,2, \cdots T\right\}$ is called an interval linguistic set, if $s_{i}$ represents an interval linguistic variable corresponding to an interval value.

In this paper we use 7-scale linguistic terms to express the interval linguistic set as below.

$$
S=\left\{\begin{array}{l}
s_{0}=A L, s_{1}=V L, s_{2}=L, s_{3}=M, \\
s_{4}=H, s_{5}=V H, s_{6}=A H
\end{array}\right\},
$$

each linguistic term in the above interval linguistic set corresponds to an interval value as listed in Table 1 .

Table 1. Linguistic terms for rating the alternatives and index weight

\begin{tabular}{|l|l|}
\hline Linguistic term & Interval value \\
\hline Absolutely high (AH) & {$[1,1]$} \\
\hline Very high (VH) & {$[0.8,1]$} \\
\hline High (H) & {$[0.6,0.8]$} \\
\hline Medium (M) & {$[0.4,0.6]$} \\
\hline Low (L) & {$[0.2,0.4]$} \\
\hline Very low (VL) & {$[0,0.2]$} \\
\hline Absolutely low (AL) & {$[0,0]$} \\
\hline
\end{tabular}

Definition 2 [4]. $\bar{a}=\left[a_{L}, a_{R}\right]$ is named a positive interval value, if $0 \leq a_{L} \leq a_{R}$. And the length of this interval is defined as length $(\bar{a})=a_{R}-a_{L}$.

Definition 3 [14]. Let $\bar{a}=\left[a_{L}, a_{R}\right], \bar{b}=\left[b_{L}, b_{R}\right]$ be two positive interval values, some basic operators are defined as

[1] $\bar{a}+\bar{b}=\left[a_{L}+b_{L}, a_{R}+b_{R}\right]$,

[2] $\lambda \bar{a}=\left[\lambda a_{L}, \lambda a_{R}\right], \forall \lambda \geq 0$,

[3] $\bar{a}^{\lambda}=\left[\left(a_{L}\right)^{\lambda},\left(a_{R}\right)^{\lambda}\right], \forall \lambda \geq 0$,

[4] $\bar{a} \times \bar{b}=\left[a_{L} \cdot b_{L}, a_{R} \cdot b_{R}\right]$.

Definition 4 [5]. Let $\bar{a}=\left[a_{L}, a_{R}\right], \bar{b}=\left[b_{L}, b_{R}\right]$ be any two positive interval values, the degree of possibility of $\bar{a} \geq \bar{b}$ is defined as

$$
P(\bar{a} \geq \bar{b})=\max \left\{1-\max \left\{\frac{b_{R}-a_{L}}{\text { length }(\bar{a})+\text { length }(\bar{b})}, 0\right\}, 0\right\} .
$$

Definition 5 [5]. Let $\left\{\bar{a}_{i}=\left[a_{i L}, a_{i R}\right] / i=1,2, \cdots, q\right\}$ be a series of interval values, a preference possibility matrix $P=\left(p_{i j}\right)_{q \times q}$ can be constructed by comparing each pair of interval values as

$$
\begin{aligned}
& p_{i j}=P\left(\bar{a}_{i} \geq \bar{a}_{j}\right) \\
& =\max \left\{1-\max \left\{\frac{a_{j R}-a_{i L}}{\text { length }\left(\bar{a}_{i}\right)+\text { length }\left(\bar{a}_{j}\right)}, 0\right\}, 0\right\},
\end{aligned}
$$

where $p_{i j} \in[0,1], \quad p_{i j}+p_{j i}=1, \quad p_{i i}=1 / 2 \quad$ for any $i, j=1,2, \cdots, q$.

Definition 6 [5] The ranking vector of all the intervals based on the preference possibility matrix is defined as $\varpi=\left(\varpi_{1}, \varpi_{2}, \cdots, \varpi_{q}\right)$ where

$$
\varpi_{i}=\frac{1}{q(q-1)}\left|\sum_{j=1}^{q} p_{i j}+\frac{q}{2}-1\right|, \quad i, j=1,2, \cdots, q .
$$

From the ranking order of $\varpi_{i}$ one can easily rank all the candidate intervals.

\section{Collaborative Group Decision Model for Internet Public Opinion Emergency with Interval Linguistic Assessment Term}

In this section, we demonstrate a collaborative decision model for internet public opinion emergency with interval linguistic assessment terms for individual and collaborative decision index. The aim of this paper focuses on how to obtain the weights of each individual and collaborative index and evaluate the severity of all the alternative internet public opinion emergencies (IPOEs) and select the appropriate decision solution. Suppose internet safety departments monitor that there are some possible emergencies $E=\left\{E_{h} / h=1,2, \cdots, q ; q \geq 2\right\}$ induced by internet public opinion. To rank all the severity of IPOEs regarding all the related emergency sections and make group decision plan, we should choose $l$ decision experts from different emergency sections as an emergency committee $\left\{D M_{1}, D M_{2}, \cdots, D M_{l}\right\}$. Suppose the decision importance of all emergency decision-makers are equal.

As we know, there are some coevolution between internet public opinion emergencies. So, each internet public opinion emergency is not only affected by some important individual indexes $I=\left\{I_{i} / i=1,2, \cdots, m\right\}$ but also affected by some collaborative decision indexes $C=\left\{C_{j} / j=1,2, \cdots, n\right\}$. And all the indexes generally have different importance degree for different emergency decision-maker in group collaborative decision process. Therefore, we assume that the linguistic weight vectors of individual indexes and collaborative indexes given by $\mathrm{k}$-th emergency decision-maker $D M_{k}$ from k-th emergency department are given by $\bar{w}_{k}=\left(\bar{w}_{1 k}, \bar{w}_{2 k}, \cdots, \bar{w}_{m k}\right)$ and $\bar{v}_{k}=\left(\bar{v}_{1 k}, \bar{v}_{2 k}, \cdots, \bar{v}_{n k}\right)$, where $\bar{w}_{i k}, \bar{v}_{j k} \in S$.

Suppose the decision-maker $D M_{k}$ evaluates internet public opinion emergencies regarding all the individual indexes as the following interval linguistic assessment matrix.

$$
\bar{D}_{k}=\left[\bar{d}_{h i k}\right]_{q \times m}=\left[\begin{array}{ll}
\bar{d}_{11 k} & \bar{d}_{12 k} \cdots \bar{d}_{1 m k} \\
\bar{d}_{21 k} & \bar{d}_{22 k} \cdots \bar{d}_{2 m k} \\
\cdots \ldots \ldots \ldots \ldots \ldots . . . \cdots & \ldots \ldots \ldots \\
\bar{d}_{q 1 k} & \bar{d}_{q 2 k} \cdots \bar{d}_{q m k}
\end{array}\right],
$$

where $\bar{d}_{h i k}$ is the interval-valued linguistic assessment information of internet public opinion emergency $E_{h}$ regarding the i-th individual index for decision-maker $D M_{k}$,

Also, suppose emergency expert $\mathrm{DM}_{k}$ evaluates all the alternative internet public opinion emergencies regarding each collaborative indexes as the following intervalvalued linguistic assessment matrix. 


$$
\bar{C}_{j k}=\left[\bar{c}_{h g j k}\right]_{q \times q}=\left[\begin{array}{l}
\bar{c}_{11 j k}, \bar{c}_{12 j k} \cdots, \bar{c}_{1 q j k} \\
\bar{c}_{21 j k}, \bar{c}_{22 j k} \cdots, \bar{c}_{2 q j k} \\
\cdots \ldots \ldots \ldots \ldots \ldots \ldots . . \cdots \cdots \\
\bar{c}_{q 1 j k}, \bar{c}_{q 2 j k} \cdots, \bar{c}_{q q j k}
\end{array}\right],
$$

where $\bar{c}_{h g j k} \in S$ is the collaborative linguistic assessment of internet public opinion emergencies $E_{h}$ and $E_{g}$ regarding the j-th collaborative index for decision-maker $D M_{k}$. Obviously, it is a symmetric matrix, and $\bar{c}_{h h j k}=[1,1], h, g=1,2, \cdots, q ; j=1,2, \cdots, n ; k=1,2, \cdots, l$.

To cope with the collaborative group decision process for IPOEs involving multiple individual and collaborative indexes, we can take the following model procedures.

Step1. Integrating all the individual index weight $\bar{w}_{i k}$ given by $D M_{k}(k=1,2, \cdots, l)$, we calculate the interval weight of the i-th individual index as

$$
\bar{w}_{i}=\frac{1}{l} \sum_{k=1}^{l} \bar{w}_{i k}=\frac{1}{l}\left[\bar{w}_{i 1}+\bar{w}_{i 2}+\cdots+\bar{w}_{i l}\right],
$$

and get the individual index weight vector $\bar{w}=\left(\bar{w}_{1}, \bar{w}_{2}, \cdots \bar{w}_{m}\right)$ of all the individual indexes of IPOEs.

Aggregate all the emergency decision-maker's interval linguistic assessment matrix $D_{k}=\left[\bar{d}_{h i k}\right]_{q \times m}$ into an overall linguistic assessment matrix $D=\left[\bar{d}_{h i}\right]_{q \times m}$, where

$$
\bar{d}_{h i}=\frac{1}{l} \sum_{k=1}^{l} \bar{d}_{h i k}=\frac{1}{l}\left[\bar{d}_{h i 1}+\bar{d}_{h i 2}+\cdots+\bar{d}_{h i l}\right] .
$$

Then we can aggregate the individual index decision matrix with weight vector and get the individual severity of each internet public opinion emergency $E_{h}$ as below.

$$
\bar{d}_{h}=\frac{1}{m}\left[\left(\bar{w}_{1} \times \bar{d}_{h_{1}}\right)+\left(\bar{w}_{2} \times \bar{d}_{h_{2}}\right)+\cdots+\left(\bar{w}_{m} \times \bar{d}_{h_{m}}\right)\right] .
$$

Step 2. Integrating all the collaborative index weight $\bar{v}_{j k}$ given by $D M_{k}$ one can get the overall collaborative index weight of the $\mathrm{j}$-th collaborative index as

$$
\bar{v}_{j}=\frac{1}{l} \sum_{k=1}^{l} \bar{v}_{j k}=\frac{1}{l}\left[\bar{v}_{j 1}+\bar{v}_{j 2}+\cdots+\bar{v}_{j l}\right],
$$

and obtain the weight vector $\bar{v}=\left(\bar{v}_{1}, \bar{v}_{2}, \cdots \bar{v}_{n}\right)$ of all the collaborative indexes of IPOE.

Aggregate all the collaborative emergency assessment matrix $\bar{C}_{j k}=\left[\bar{c}_{h g j k}\right]_{q \times q}$ of $\mathrm{j}$-th collaborative index for all the emergency decision-maker $k(k=1,2, \cdots, l)$ into an overall collaborative emergency assessment matrix $\bar{C}_{j}=\left[\bar{c}_{h g j}\right]_{q \times q}$ regarding $\mathrm{j}$-th collaborative index, where

$$
\bar{c}_{h g j}=\frac{1}{l} \sum_{k=1}^{l} \bar{c}_{h g j k}=\frac{1}{l}\left[\bar{c}_{h g j 1}+\bar{c}_{h g j 2}+\cdots+\bar{c}_{h g j l}\right],
$$

Then we can calculate the collaborative severity of IPOE $E_{h}$ regarding $E_{g}$ by aggregating the collaborative emergency assessment $\bar{c}_{h g j}$ with weight vector $\bar{v}_{j}$ below.

$$
\begin{aligned}
& \bar{c}_{h g}=\frac{1}{n} \sum_{j=1}^{n} \bar{v}_{j} \bar{c}_{h g j} \\
& =\frac{1}{n}\left[\left(\bar{v}_{1} \times \bar{c}_{h g 1}\right)+\left(\bar{v}_{2} \times \bar{c}_{h g 2}\right)+\cdots+\left(\bar{v}_{n} \times \bar{c}_{h g n}\right)\right] .
\end{aligned}
$$

Finally, we calculate the collective collaboration severity value of each internet public opinion emergency $E_{h}$ by aggregating all the collaborative severity of $E_{h}$ with other internet public opinion emergencies $E_{g}(g=1,2, \cdots, q)$ as follows.

$$
\bar{c}_{h}=\frac{1}{q} \sum_{g=1}^{q} \bar{c}_{h g}=\frac{1}{q}\left[\bar{c}_{h 1}+\bar{c}_{h 2}+\cdots+\bar{c}_{h q}\right] .
$$

Step 3. Consider all the emergency decision-makers possibly assign different weight $\lambda$ to individual severity and $1-\lambda$ to collaborative severity for each internet public opinion emergency $E_{h}$, we calculate the overall crisis severity of each IPOE $E_{h}$ by combining individual severity and collaborative severity as

$$
\bar{\phi}_{h}=\lambda \bar{d}_{h}+(1-\lambda) \bar{c}_{h}, \forall h=1,2, \cdots, q .
$$

Step 4. Compute the crisis preference possibility matrix $P=\left(p_{h g}\right)_{q \times q}$ between all the overall crisis interval values $\bar{\phi}_{h}(h=1,2, \cdots, q)$ of internet public opinion emergencies, where $p_{h g}=P\left(\bar{\phi}_{h} \geq \bar{\phi}_{g}\right)$ is the crisis preference degree of IPOE $E_{h}$ to $E_{g}$.

Step 5. We use $\varpi_{i}=\frac{1}{q(q-1)}\left|\sum_{j=1}^{q} p_{i j}+\frac{q}{2}-1\right|$ to calculate the ranking vector $\varpi=\left(\varpi_{1}, \varpi_{2}, \cdots, \varpi_{q}\right)$ of all the crisis severity intervals $\bar{\phi}_{h}(h=1,2, \cdots, q)$ of IPOES according to the crisis preference possibility matrix $P=\left(p_{h g}\right)_{q \times q}$. From the ranking order of $\varpi_{i}$ one can easily rank all the crisis interval $\bar{\phi}_{h}$ of internet public opinion emergency $E_{h}(h=1,2, \cdots, q)$ according to the ascending order of $\varpi_{h}(h=1,2, \cdots, q)$. The larger is $\varpi_{i}$, the more severe is the internet public opinion emergency $E_{i}$. Thus, the emergency department can dispose of most severe internet public opinion emergencies according to the ranking vector in time.

\section{Illustrative Example}

Suppose that security monitoring departments collect internet public opinions from internet news and micro-blogs, and find that four internet public opinion emergencies $E_{1}, E_{2}, E_{3} E_{4}$ will possible take place in the city. Due to the shortage of urban emergency personnel and facilities, the most serious internet public opinion emergency should be disposed firstly to avoid its evolution to extreme emergency. Therefore, it requires government to combine some related emergency departments to make group decision for all the internet 
public opinion emergencies. Now we choose four emergency experts from different emergency departments including network supervision department, governmental administration department, public security department and medical department as a decision committee to evaluate the comprehensive crisis of each emergency and make group decision.

Since the internet public opinion emergencies outbreak suddenly, it is difficult for the emergency decision experts to get accurate index data of internet public opinion emergencies in a short time. Therefore, in order to make efficient emergency group decision, the emergency experts often employ some interval linguistic terms to assess emergency individual indexes including the attention degree of internet sentiment $\left(I_{1}\right)$, the tendency of public topic opinion $\left(I_{2}\right)$, the diffusion rate of event $\left(I_{3}\right)$, and the economic damage degree of emergency $\left(I_{4}\right)$. The above uncertain linguistic term assessment can be easily expressed by some interval values as listed in Table 1.

Also we consider the regional similarity $\left(c_{1}\right)$, the source similarity of internet public sentiment $\left(c_{2}\right)$, and the coordinated evolution of event derivatives $\left(c_{3}\right)$ as collaborative indexes of internet public opinion emergency. Assume that the index weight information of individual index and collaborative index assigned by four emergency experts from different emergency sections are listed in the following Table 2.

Table 2. Interval linguistic weight information of individual index and collaborative indexes

\begin{tabular}{|c|c|c|c|c|c|c|c|c|}
\hline \multirow{2}{*}{ Decision-maker } & \multicolumn{5}{|c|}{ Individual index } & \multicolumn{5}{|c|}{ Collaborative index } \\
\cline { 2 - 10 } & I1 & I2 & I3 & I4 & C1 & C2 & C3 & C4 \\
\hline DM1 & AH & VH & H & M & VH & H & VH & VH \\
\hline DM2 & H & M & VH & VH & AH & VH & H & H \\
\hline DM3 & VH & VH & M & H & H & AH & VH & H \\
\hline DM4 & VH & M & H & AH & AH & H & H & VH \\
\hline
\end{tabular}

Below we also list the interval linguistic assessment information of individual indexes and collaborative indexes of internet public opinion emergencies for each emergency decision-maker in Table 3 - Table 7.

Table 3. Linguistic assessment information of individual index for IPOE

\begin{tabular}{|c|c|c|c|c|c|}
\hline \multirow{3}{*}{ Decision-maker } & $\begin{array}{c}\text { Emergency } \\
\text { events }\end{array}$ & \multicolumn{4}{|c|}{$\begin{array}{c}\text { Linguistic assessment for individual } \\
\text { index of emergency events }\end{array}$} \\
\cline { 2 - 6 } & $\mathrm{I}$ & $\mathrm{I} 2$ & $\mathrm{I}$ & $\mathrm{I}$ \\
\hline \multirow{4}{*}{ DM1 } & $\mathrm{M}$ & $\mathrm{VH}$ & $\mathrm{M}$ & $\mathrm{H}$ \\
\cline { 2 - 6 } & $\mathrm{E} 2$ & $\mathrm{AL}$ & $\mathrm{H}$ & $\mathrm{M}$ & $\mathrm{VH}$ \\
\cline { 2 - 6 } & $\mathrm{E} 3$ & $\mathrm{~L}$ & $\mathrm{M}$ & $\mathrm{AH}$ & $\mathrm{H}$ \\
\cline { 2 - 6 } & $\mathrm{E} 4$ & $\mathrm{VL}$ & $\mathrm{L}$ & $\mathrm{H}$ & $\mathrm{M}$ \\
\hline \multirow{4}{*}{ DM2 } & $\mathrm{E} 1$ & $\mathrm{~L}$ & $\mathrm{VH}$ & $\mathrm{L}$ & $\mathrm{M}$ \\
\cline { 2 - 6 } & $\mathrm{E} 2$ & $\mathrm{H}$ & $\mathrm{M}$ & $\mathrm{VL}$ & $\mathrm{VH}$ \\
\cline { 2 - 6 } & $\mathrm{E} 3$ & $\mathrm{M}$ & $\mathrm{VH}$ & $\mathrm{H}$ & $\mathrm{M}$ \\
\cline { 2 - 6 } & $\mathrm{E} 4$ & $\mathrm{~L}$ & $\mathrm{H}$ & $\mathrm{M}$ & $\mathrm{VH}$ \\
\hline \multirow{4}{*}{ DM3 } & $\mathrm{E} 1$ & $\mathrm{~L}$ & $\mathrm{AH}$ & $\mathrm{H}$ & $\mathrm{M}$ \\
\cline { 2 - 6 } & $\mathrm{E} 2$ & $\mathrm{H}$ & $\mathrm{VH}$ & $\mathrm{L}$ & $\mathrm{H}$ \\
\cline { 2 - 6 } & $\mathrm{E} 3$ & $\mathrm{~L}$ & $\mathrm{H}$ & $\mathrm{VH}$ & $\mathrm{H}$ \\
\cline { 2 - 6 } & $\mathrm{E} 4$ & $\mathrm{H}$ & $\mathrm{M}$ & $\mathrm{H}$ & $\mathrm{L}$ \\
\hline \multirow{5}{*}{ DM4 } & $\mathrm{E} 1$ & $\mathrm{M}$ & $\mathrm{L}$ & $\mathrm{VH}$ & $\mathrm{H}$ \\
\cline { 2 - 6 } & $\mathrm{E} 2$ & $\mathrm{~L}$ & $\mathrm{M}$ & $\mathrm{H}$ & $\mathrm{VH}$ \\
\cline { 2 - 6 } & $\mathrm{E} 3$ & $\mathrm{H}$ & $\mathrm{VH}$ & $\mathrm{M}$ & $\mathrm{L}$ \\
\cline { 2 - 6 } & $\mathrm{E} 4$ & $\mathrm{VH}$ & $\mathrm{L}$ & $\mathrm{AH}$ & $\mathrm{M}$ \\
\hline
\end{tabular}

Table 4. Linguistic assessment of collaborative emergency index for DM1

\begin{tabular}{|c|c|c|c|c|c|}
\hline $\begin{array}{c}\text { Collaborative } \\
\text { index }\end{array}$ & $\begin{array}{c}\text { Emergency } \\
\text { events }\end{array}$ & \multicolumn{4}{|c|}{ Emergency events } \\
\cline { 3 - 6 } & $\mathrm{E} 1$ & $\mathrm{E} 2$ & $\mathrm{E} 3$ & $\mathrm{E}$ \\
\hline \multirow{4}{*}{$\mathrm{C} 1$} & $\mathrm{E} 1$ & $\mathrm{AH}$ & $\mathrm{M}$ & $\mathrm{VL}$ & $\mathrm{L}$ \\
\cline { 2 - 6 } & $\mathrm{E} 2$ & $\mathrm{M}$ & $\mathrm{AH}$ & $\mathrm{M}$ & $\mathrm{VL}$ \\
\cline { 2 - 6 } & $\mathrm{E} 3$ & $\mathrm{H}$ & $\mathrm{H}$ & $\mathrm{AH}$ & $\mathrm{M}$ \\
\cline { 2 - 6 } & $\mathrm{E} 4$ & $\mathrm{VL}$ & $\mathrm{M}$ & $\mathrm{M}$ & $\mathrm{AH}$ \\
\hline \multirow{4}{*}{ C2 } & $\mathrm{E} 1$ & $\mathrm{AH}$ & $\mathrm{L}$ & $\mathrm{M}$ & $\mathrm{H}$ \\
\cline { 2 - 6 } & $\mathrm{E} 2$ & $\mathrm{~L}$ & $\mathrm{AH}$ & $\mathrm{H}$ & $\mathrm{VL}$ \\
\cline { 2 - 6 } & $\mathrm{E} 3$ & $\mathrm{H}$ & $\mathrm{M}$ & $\mathrm{AH}$ & $\mathrm{L}$ \\
\cline { 2 - 6 } & $\mathrm{E} 4$ & $\mathrm{M}$ & $\mathrm{H}$ & $\mathrm{M}$ & $\mathrm{AH}$ \\
\hline \multirow{4}{*}{$\mathrm{C} 3$} & $\mathrm{E} 1$ & $\mathrm{AH}$ & $\mathrm{H}$ & $\mathrm{L}$ & $\mathrm{L}$ \\
\cline { 2 - 6 } & $\mathrm{E} 2$ & $\mathrm{H}$ & $\mathrm{AH}$ & $\mathrm{AL}$ & $\mathrm{M}$ \\
\cline { 2 - 6 } & $\mathrm{E} 3$ & $\mathrm{M}$ & $\mathrm{L}$ & $\mathrm{AH}$ & $\mathrm{M}$ \\
\cline { 2 - 6 } & $\mathrm{E} 4$ & $\mathrm{~L}$ & $\mathrm{H}$ & $\mathrm{M}$ & $\mathrm{AH}$ \\
\hline
\end{tabular}

Table 5. Linguistic assessment of collaborative emergency index for DM2

\begin{tabular}{|c|c|c|c|c|c|}
\hline \multirow{2}{*}{ Collaborative index } & \multirow{2}{*}{ Emergency events } & \multicolumn{4}{|c|}{ Emergency events } \\
\cline { 2 - 6 } & & E1 & E2 & E3 & E4 \\
\hline \multirow{3}{*}{ C1 } & E1 & AH & M & H & L \\
\cline { 2 - 6 } & E2 & VH & AH & M & VL \\
\cline { 2 - 6 } & E3 & H & L & AH & L \\
\cline { 2 - 6 } & E4 & VL & M & VH & AH \\
\hline \multirow{4}{*}{ C2 } & E1 & AH & VL & M & VH \\
\cline { 2 - 6 } & E2 & VL & AH & VH & L \\
\cline { 2 - 6 } & E3 & M & M & AH & L \\
\cline { 2 - 6 } & E4 & L & VH & M & AH \\
\hline \multirow{3}{*}{ C3 } & E1 & AH & H & L & VL \\
\cline { 2 - 6 } & E2 & VH & AH & VL & M \\
\cline { 2 - 6 } & E3 & L & M & AH & M \\
\cline { 2 - 6 } & E4 & VL & VH & M & AH \\
\hline
\end{tabular}

Table 6. Linguistic assessment of collaborative emergency index for DM3

\begin{tabular}{|c|c|c|c|c|c|}
\hline \multirow{3}{*}{ Collaborative index } & \multirow{2}{*}{ Emergency events } & \multicolumn{4}{|c|}{ Emergency events } \\
\cline { 2 - 6 } & & E1 & E2 & E3 & E4 \\
\hline \multirow{3}{*}{ C1 } & E1 & AH & M & VH & M \\
\cline { 2 - 6 } & E2 & M & AH & AL & VH \\
\cline { 2 - 6 } & E3 & VH & AL & AH & L \\
\cline { 2 - 6 } & E4 & L & MH & H & AH \\
\hline \multirow{4}{*}{ C2 } & E1 & AH & AL & H & VL \\
\cline { 2 - 6 } & E2 & AL & AH & VH & L \\
\cline { 2 - 6 } & E3 & H & VH & AH & L \\
\cline { 2 - 6 } & E4 & VL & H & M & AH \\
\hline \multirow{4}{*}{ C3 } & E1 & AH & H & M & VL \\
\cline { 2 - 6 } & E2 & H & AH & M & VH \\
\cline { 2 - 6 } & E3 & M & H & AH & H \\
\cline { 2 - 6 } & E4 & VL & L & H & AH \\
\hline
\end{tabular}

Table 7. Linguistic assessment of collaborative emergency index for DM4

\begin{tabular}{|c|c|c|c|c|c|}
\hline $\begin{array}{c}\text { Collaborative } \\
\text { index }\end{array}$ & $\begin{array}{c}\text { Emergency } \\
\text { events }\end{array}$ & \multicolumn{4}{|c|}{ Emergency events } \\
\cline { 3 - 6 } & $\mathrm{E} 1$ & $\mathrm{E} 2$ & $\mathrm{E} 3$ & $\mathrm{E} 4$ \\
\hline \multirow{4}{*}{$\mathrm{C} 1$} & $\mathrm{E} 1$ & $\mathrm{AH}$ & $\mathrm{L}$ & $\mathrm{VH}$ & $\mathrm{VL}$ \\
\cline { 2 - 6 } & $\mathrm{E} 2$ & $\mathrm{~L}$ & $\mathrm{AH}$ & $\mathrm{M}$ & $\mathrm{VH}$ \\
\cline { 2 - 6 } & $\mathrm{E} 3$ & $\mathrm{VL}$ & $\mathrm{H}$ & $\mathrm{AH}$ & $\mathrm{H}$ \\
\cline { 2 - 6 } & $\mathrm{E} 4$ & $\mathrm{M}$ & $\mathrm{H}$ & $\mathrm{VH}$ & $\mathrm{AH}$ \\
\hline \multirow{4}{*}{ C2 } & $\mathrm{E} 1$ & $\mathrm{AH}$ & $\mathrm{H}$ & $\mathrm{VH}$ & $\mathrm{L}$ \\
\cline { 2 - 6 } & $\mathrm{E} 2$ & $\mathrm{H}$ & $\mathrm{AH}$ & $\mathrm{L}$ & $\mathrm{VL}$ \\
\cline { 2 - 6 } & $\mathrm{E} 3$ & $\mathrm{VH}$ & $\mathrm{L}$ & $\mathrm{AH}$ & $\mathrm{M}$ \\
\cline { 2 - 6 } & $\mathrm{E} 4$ & $\mathrm{M}$ & $\mathrm{VL}$ & $\mathrm{VH}$ & $\mathrm{AH}$ \\
\hline \multirow{4}{*}{$\mathrm{C} 3$} & $\mathrm{E} 1$ & $\mathrm{AH}$ & $\mathrm{H}$ & $\mathrm{VH}$ & $\mathrm{AL}$ \\
\cline { 2 - 6 } & $\mathrm{E} 2$ & $\mathrm{H}$ & $\mathrm{AH}$ & $\mathrm{H}$ & $\mathrm{L}$ \\
\cline { 2 - 6 } & $\mathrm{E} 3$ & $\mathrm{VH}$ & $\mathrm{H}$ & $\mathrm{AH}$ & $\mathrm{M}$ \\
\cline { 2 - 6 } & $\mathrm{E} 4$ & $\mathrm{M}$ & $\mathrm{VL}$ & $\mathrm{VVH}$ & $\mathrm{AH}$ \\
\hline
\end{tabular}


To make group decision according to the assessed individual and collaborative indexes of IPOEs, we first transfer the linguistic assessment terms in above Table 2 Table 7 to the corresponding interval values as in Table1. According to Table 2 and formula (1),

$$
\bar{w}_{i}=\frac{1}{4}\left[\bar{w}_{i 1}+\bar{w}_{i 2}+\bar{w}_{i 3}+\bar{w}_{i 4}\right], i=1,2,3,4 .
$$

we compute the weight of individual index of IPOE as

$$
\begin{aligned}
& \bar{w}_{1}=[0.8,0.95], \bar{w}_{2}=[0.6,0.8], \\
& \bar{w}_{3}=[0.6,0.8], \bar{w}_{4}=[0.7,0.85] .
\end{aligned}
$$

Based on formula(4), $\bar{v}_{j}=\frac{1}{4}\left[\bar{v}_{j 1}+\bar{v}_{j 2}+\bar{v}_{j 3}+\bar{v}_{j 4}\right]$, $j=1,2,3$, we can calculate the interval weight of each collaborative index of IPOE as

$$
\bar{v}_{1}=[0.85,0.95], \bar{v}_{2}=[0.75,0.9], \bar{v}_{3}=[0.7,0.9] \text {. }
$$

Also, by employing formula (2) (3) we calculate the individual severity $\bar{d}_{h}$ of each internet public opinion emergency $E_{h}$ as listed in the following Table 8.

Table 8. Individual interval-valued performance assessment of emergency

\begin{tabular}{|c|c|c|c|c|c|}
\hline$E_{h}$ & $\bar{d}_{h 1}$ & $\bar{d}_{h 2}$ & $\bar{d}_{h 3}$ & $\bar{d}_{h 4}$ & $\bar{d}_{h}$ \\
\hline E1 & {$[0.3,0.5]$} & {$[0.7,0.85]$} & {$[0.5,0.7]$} & {$[0.5,0.7]$} & $\begin{array}{c}{[0.3275,} \\
0.5775]\end{array}$ \\
\hline E2 & {$[0.45,0.5]$} & {$[0.55,0.75]$} & {$[0.3,0.5]$} & {$[0.75,0.95]$} & $\begin{array}{c}{[0.34875,} \\
0.569375]\end{array}$ \\
\hline E3 & {$[0.35,0.55]$} & {$[0.65,0.85]$} & {$[0.7,0.85]$} & {$[0.45,0.65]$} & $\begin{array}{c}{[0.35125,} \\
0.60875]\end{array}$ \\
\hline E4 & {$[0.4,0.6]$} & {$[0.35,0.55]$} & {$[0.65,0.8]$} & {$[0.45,0.65]$} & $\begin{array}{c}{[0.30875,} \\
0.550625]\end{array}$ \\
\hline
\end{tabular}

By utilizing formula (5) and (6) we can compute the collaborative severity of the internet public opinion emergencies as listed in Table 9.

Table 9. Collaborative interval-valued performance assessment of emergency

\begin{tabular}{|c|c|c|}
\hline Collaboration & E1 & E2 \\
\hline E1 & {$[0.766,0.9166]$} & {$[0.28916,0.51916]$} \\
\hline E2 & {$[0.32916,0.56583]$} & {$[0.766,0.9166]$} \\
\hline E3 & {$[0.3966,0.6566]$} & {$[0.3166,0.54833]$} \\
\hline E4 & {$[0.14,0.35083]$} & {$[0.345833,0.595833]$} \\
\hline Collaboration & E3 & E4 \\
\hline E1 & {$[0.3866,0.6425]$} & {$[0.16833,0.366]$} \\
\hline E2 & {$[0.2933,0.5775]$} & {$[0.1966,0.415]$} \\
\hline E3 & {$[0.766,0.9166]$} & {$[0.266,0.50416]$} \\
\hline E4 & {$[0.45166,0.735]$} & {$[0.766,0.9166]$} \\
\hline
\end{tabular}

By using formula (7) one can calculate the overall collaborative crisis severity of each internet public opinion emergency as follows.

$$
\begin{gathered}
\bar{c}_{1}=\frac{1}{4}\left[\bar{c}_{11}+\bar{c}_{12}+\bar{c}_{13}+\bar{c}_{14}\right]=[0.4027025,0.611245] ; \\
\bar{c}_{2}=\frac{1}{4}\left[\bar{c}_{21}+\bar{c}_{22}+\bar{c}_{23}+\bar{c}_{24}\right]=[0.39645,0.60375] ; \\
\bar{c}_{3}=\frac{1}{4}\left[\bar{c}_{31}+\bar{c}_{32}+\bar{c}_{33}+\bar{c}_{34}\right]=[0.43666,0.65645] ; \\
\bar{c}_{4}=\frac{1}{4}\left[\bar{c}_{41}+\bar{c}_{42}+\bar{c}_{43}+\bar{c}_{44}\right]=[0.426,0.6496] .
\end{gathered}
$$

Suppose all the emergency decision-makers assign weight $\lambda=0.6$ to individual crisis and $1-\lambda=0.4$ to collaborative crisis, by using formula (8) we can aggregate the individual crisis and collaborative crisis to get the overall crisis of each internet public opinion emergency $E_{h}(h=1,2,3,4)$ as below.

$$
\begin{gathered}
\bar{\phi}_{h}=\lambda \bar{d}_{h}+(1-\lambda) \bar{c}_{h}=0.6 \bar{d}_{h}+0.4 \bar{c}_{h}, h=1,2,3,4 . \\
\bar{\phi}_{1}=[0.35758,0.591], \bar{\phi}_{2}=[0.36783,0.583125], \\
\bar{\phi}_{3}=[0.385414,0.62783], \bar{\phi}_{4}=[0.35565,0.590215] .
\end{gathered}
$$

Thus, we compute the following crisis preference possibility matrix between the overall crisis intervals $\bar{\phi}_{h}(h=1,2, \cdots, 4)$ of all the IPOEs below.

$$
P=\left(p_{h g}\right)_{4 \times 4}=\left(\begin{array}{lcccc}
0.5 & 0.4973 & 0.43200 & 0.5029 \\
0.5027 & 0.5 & 0.43196 & 0.50566 \\
0.568 & 0.56804 & 0.50 & 0.5706 \\
0.4971 & 0.49434 & 0.4294 & 0.5
\end{array}\right)
$$

where $p_{h g}=P\left(\bar{\phi}_{h} \geq \bar{\phi}_{g}\right), h, g=1,2,3,4$.

According to the above crisis preference possibility matrix we calculate the ranking vector of all the crisis interval values of internet public opinion emergencies as

$$
\begin{aligned}
& \omega=\left(\varpi_{1}, \varpi_{2}, \varpi_{3}, \varpi_{4}\right) \\
& =(0.24435,0.245027,0.26722,0.2434),
\end{aligned}
$$

where $\varpi_{i}=\frac{1}{4(4-1)}\left|\sum_{j=1}^{4} p_{i j}+\frac{4}{2}-1\right|, i=1,2,3,4$.

From the order of $\varpi_{i}$ one can easily rank all the crisis interval values $\bar{\phi}_{h}(h=1,2,3,4)$, then we get the ranking of all the overall crisis severity of internet public opinion emergencies as $\bar{\phi}_{3} \succ \bar{\phi}_{2} \succ \bar{\phi}_{1} \succ \bar{\phi}_{4}$ according to the ascending order of $\varpi_{i}$. The emergency department should dispose of the most severe emergency $E_{3}$ according to the above ranking vector. Thus, the top emergency decision department should deal with all the IPOEs according to the crisis severity ranking $E_{3} \succ E_{2} \succ E_{1} \succ E_{4}$ due to the limited emergency resources and emergency equipments.

\section{Conclusion}

This paper proposes a new group decision method for internet public opinion emergency with interval-valued linguistic assessments involved multiple emergency decision experts. By utilizing the proposed collaborative emergency group decision model we can easily calculate the overall crisis severity of each IPOE. According to the ranking of crisis severity, the top emergency department can effectively handle all the internet public opinion emergencies and take the corresponding emergency plan to execute emergency operation in order. 


\section{Acknowledgements}

This paper is supported by the Natural Science Foundation of Guangdong Province, China under Grant 2017A030313435 and 2018A030313996.

\section{References}

[1] Li ,Y.T., Liu,Y., "Study on the grey early warning evaluation of network public sentiment," Journal of Intelligence, 30(4), 24-27, 2011.

[2] Zhang, Y.W., Qi, Y.J., "The interaction mechanism between network public sentiment and abnormal emergency," Journal of Informatics, 9, 1-6, 2010.

[3] Xu,Z.S, Da,Q.L., "New Method for Interval multi-attribute decision-making," Journal of Southeast University, 33(4), 498-501, 2003.

[4] Xu, Z.S., "A practical method for priority of interval number complementary judgment matrix," Operations Research and Management Science, 10, 16-19,2001.

[5] Xu, Z.S., D,Q.L., "Multi-attribute decision-making based on fuzzy linguistic assessments," Journal of Southeast University, 32(4), 656-658, 2002.

[6] Bai, C. Z., Zhang, R., Shen, S. , Huang, C.F., Fan, X., "Intervalvalued probabilistic linguistic term sets in multi-criteria group decision making," International Journal of Intelligent Systems, 33(6), 1301-1321, 2018.

[7] Zhang, Z. X., Wang,L., Wang, Y.M. , “An Emergency Decision Making Method Based on Prospect Theory for Different Emergency Situations," International Journal of Disaster Risk Science, 9 (3), 407-420, 2018.
[8] Liu, C., Qian, J., Guo, D. H., Liu, Y., “A Spatio-temporal Scenario Model for Emergency Decision," GeoInformatica, 22 (2), 411-433, 2018.

[9] Yu,Y., Guo, D., "Efficient CPS model based online opinion governance modeling and evaluation for emergency accidents," Geo Informatica, 22(2), 479-502, 2018.

[10] Wu, W.S., Kou, G., Peng, Y., Shi, Y., “A fuzzy multi-criteria emergency decision-making method," Systems EngineeringTheory and Practice, 32(6), 1298-1304, 2012.

[11] Zhang, Z.X., Wang,L., Rodríguez. R. M., Wang. Y.M., L. Martínez. "A hesitant group emergency decision making method based on prospect theory," Complex Intell. Syst., 3, 177-187, 2017.

[12] Jing, K., Zhao, X.N., Wang,Y. M., "Local group decision making for emergency under uncertainty based on interval preference," Operations Research and Management Science, 19(2), 97-103, 2010.

[13] Tang, R., Wang, H. Y., Ma, S. J., "Study on group decision model of extreme flood disaster emergency in city," Forecasting, 31(3), 71-75, 2012.

[14] Chen, T.Y., Tsao, C.Y., "The interval-valued fuzzy TOPSIS method and experimental analysis," Fuzzy Sets and Systems, 159, 1410-1428, 2008.

[15] Xu, Z.S., "Consistency of interval fuzzy preference relations in group decision making," Applied Soft Computing, 11(5), 3898-3909, 2011.

[16] Tapia Garc, J.M. , del Moral, M.J., Martínez, M.A. , et al., "A consensus model for group decision making problems with linguistic interval fuzzy preference relations," Expert Systems with Applications ,39(11), 10022-10030, 2012.

[17] $\mathrm{Yu}, \mathrm{L} .$, Lai, K. K., "A distance-based group decision-making methodology for multi-person multi-criteria emergency decision support," Decision Support Systems, 51, 307-315, 2011.

[18] Chen, X., Wang, Y., Wu, L.Y., Yan, G.Y., Zhu, W., "Emergency decision model with multiple stages, multiple objectives and multi-divisional cooperation," Systems Engineering-Theory and Practice, 30(11), 1977-1985, 2010.

C The Author(s) 2019. This article is an open access article distributed under the terms and conditions of the Creative Commons Attribution (CC BY) license (http://creativecommons.org/licenses/by/4.0/). 